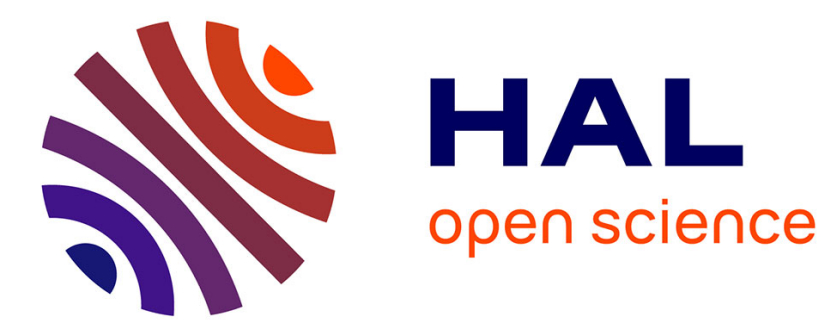

\title{
Les leçons et traités d'astronomie populaire dans le premier XIXe siècle
}

\author{
Carole Christen
}

\section{To cite this version:}

Carole Christen. Les leçons et traités d'astronomie populaire dans le premier XIXe siècle. Romantisme: la revue du dix-neuvième siècle, 2014, 166 (4), 10.3917/rom.166.0008 . hal-01700558

\section{HAL Id: hal-01700558 \\ https://hal.science/hal-01700558}

Submitted on 7 Feb 2018

HAL is a multi-disciplinary open access archive for the deposit and dissemination of scientific research documents, whether they are published or not. The documents may come from teaching and research institutions in France or abroad, or from public or private research centers.
L'archive ouverte pluridisciplinaire HAL, est destinée au dépôt et à la diffusion de documents scientifiques de niveau recherche, publiés ou non, émanant des établissements d'enseignement et de recherche français ou étrangers, des laboratoires publics ou privés. 


\section{LES LEÇONS ET TRAITÉS D'ASTRONOMIE POPULAIRE DANS LE PREMIER XIX ${ }^{E}$ SIĖCLE}

Carole Christen

Armand Colin | «Romantisme »

$2014 / 4 n^{\circ} 166$ | pages 8 à 20

ISSN 0048-8593

ISBN 9782200929091

Article disponible en ligne à l'adresse :

https://www.cairn.info/revue-romantisme-2014-4-page-8.htm

\section{Pour citer cet article :}

Carole Christen, « Les leçons et traités d'astronomie populaire dans le premier XIX siècle », Romantisme 2014/4 ( $\left.\mathrm{n}^{\circ} 166\right)$, p. 8-20.

DOI 10.3917/rom.166.0008

Distribution électronique Cairn.info pour Armand Colin.

(C) Armand Colin. Tous droits réservés pour tous pays.

La reproduction ou représentation de cet article, notamment par photocopie, n'est autorisée que dans les limites des conditions générales d'utilisation du site ou, le cas échéant, des conditions générales de la licence souscrite par votre établissement. Toute autre reproduction ou représentation, en tout ou partie, sous quelque forme et de quelque manière que ce soit, est interdite sauf accord préalable et écrit de l'éditeur, en dehors des cas prévus par la législation en vigueur en France. Il est précisé que son stockage dans une base de données est également interdit. 


\section{Les leçons et traités d'astronomie populaire dans le premier XIX ${ }^{e}$ siècle}

Héritée des Lumières et de la Révolution française, la question de l'éducation, de l'instruction et de l'enseignement du peuple, à savoir la classe la plus nombreuse et la plus pauvre, devient centrale dans le premier XIX ${ }^{\mathrm{e}}$ siècle. L'adjectif " populaire " traduit désormais les valeurs du discours progressiste. Carnot, dans un rapport à Napoléon, présente les promoteurs de la méthode mutuelle dans l'enseignement comme les " créateurs et directeurs de l'éducation populaire " ${ }^{1}$ et Guizot, dans ses mémoires aborde la période en évoquant le mouvement en faveur de ce qu'il appelle à son tour « l'instruction populaire ${ }^{2}$ ». L'adjectif " populaire » est également accolé aux livres, aux bibliothèques, aux almanachs, à la science ${ }^{3}$ et en particulier à l'astronomie comme l'attestent la publication en $1844 \mathrm{du}$ Traitéphilosophique d'astronomie populaire d'Auguste Comte et, celle dix ans plus tard, de l'Astronomie populaire œuvre posthume de François Arago. Ces deux ouvrages sont issus des cours publics gratuits qu'Arago et Comte ont donnés : le premier à l'Observatoire dès 1813 et le second au sein de l'Association polytechnique fondée aux lendemains de la Révolution de 1830 pour diffuser l'instruction gratuite et publique des ouvriers. Alexandre de Humboldt, ami d'Arago qui a insisté pour qu'il publie son cours, ne souhaitait pas ce terme de " populaire » dans le titre comme il lui écrit en août 1850 : «Ton Astronomie que je demande en grâce de ne pas nommer populaire, mot dont on a tant abusé comme du mot socialiste ${ }^{4}$ ». Au contraire, Jean-Augustin Barral, élève et ami d'Arago auquel il a confié toutes les notes de ses cours et qui a publié cet ouvrage entre 1854 et 1857 , insiste sur sa dimension " populaire ": "Savants, industriels, agriculteurs, hommes du monde, Arago a travaillé pour tous. À la gloire de découvertes fécondes en résultats pratiques, il a joint l'imparable faculté de rendre accessibles à toutes les intelligences les connaissances scientifiques les plus élevées, en un mot, de rendre la science populaire ${ }^{5}$. " Que faut-il entendre par « astronomie populaire » ? Y-a-t-il équivalence

1. Cité par François Jacquet-Francillon, Naissances de l'école du peuple, 1815-1870, Paris, Les Éditions de 1'Atelier, 1995, p. 90-91.

2. Ibid.

3. Bernadette Bensaude-Vincent, La science contre l'opinion. Histoire d'un divorce, Paris, Les Empêcheurs de penser en rond, 2003, voir en particulier le chapitre «1835, science officielle et science populaire », p. 87-116.

4. Correspondance d'Alexandre de Humboldt avec François Arago (1809-1853), publiée par ErnestThéodore Hamy, Paris, E. Guilmoto, 1908.

5. François Arago, Astronomie populaire, Paris. L. Guérin, 1967 ( $2^{\mathrm{e}}$ éd.), t. 4, Prospectus en fin d'ouvrage. 
entre " populaire » et " peuple ${ }^{6}$ " ? Cette astronomie est-elle populaire parce qu'elle est destinée au peuple, en particulier les petits artisans et ouvriers parisiens, ou parce qu'elle est facile à comprendre, distrayante, voire anti-académique privilégiant la figure de l'amateur de science et appartenant à la bourgeoisie ? Y-a-t-il un réel souci de communion entre le savant républicain et le peuple autour de la connaissance scientifique, et par là un projet politique d'émancipation du peuple grâce au savoir astronomique qui se dégagerait ? La contextualisation de la création de ces cours et l'analyse du contenu de ces leçons et autres traités élémentaires d'astronomie qui paraissent à la fin de la Restauration et sous la monarchie de Juillet, avant donc que s'affirme dans le second XIX ${ }^{\mathrm{e}}$ siècle le grand mouvement de vulgarisation scientifique - «la science pour tous ${ }^{7}$ " - conduisent à s'interroger, d'une part sur la façon dont les savants cherchent à rendre populaire une science aussi élaborée et mathématisée que l'astronomie dans ce premier XIX ${ }^{\mathrm{e}}$ siècle et, d'autre part, sur l'usage et l'utilité pour le " peuple »-que doit-il apprendre du Ciel ? - que ces savants accordent à l'astronomie. On tentera d'approcher le public qui suit ces cours et lit ces traités. Le cours d'Arago et celui de Comte seront successivement étudiés. La place de l'astronomie dans les bibliothèques populaires et plus généralement dans l'éducation du peuple ${ }^{8}$ sera ensuite analysée.

\section{ARAGO À L'OBSERVATOIRE : UN COURS D'ASTRONOMIE POPULAIRE OU UN COURS POPULAIRE D'ASTRONOMIE?}

En février 1813, François Arago, polytechnicien, secrétaire du Bureau des Longitudes, membre de l'Académie des Sciences depuis 1809, reprend le cours public d'astronomie que le Bureau des Longitudes doit délivrer selon la loi du 8 messidor an III (1795) qui a créé cet organisme. Le but de ce cours est de former des jeunes gens, futurs directeurs des observatoires de province ou astronomes des

6. Clifford D. Conner, Histoire populaire des sciences, Paris, Points Sciences, 2014 (1 ${ }^{\text {re }}$ éd. à NewYork en 2005 traduit en français en 2011), voir le chapitre 1 : «Quelles sciences ? Quelle histoire ? Quel peuple ?», p. 19-49.

7. Voir Bruno Béguet (dir.), La science pour tous. Sur la vulgarisation scientifique en France de 1850 à 1914, Paris, Bibliothèque du CNAM, 1990 ; Daniel Raichvarg, Jean Jacques, Savants et ignorants. Une histoire de la vulgarisation des sciences, Paris, Éditions du Seuil, 1991 ; «Sciences pour tous », Romantisme, $\mathrm{n}^{\circ}$ 65, 1989 ; Bernadette Bensaude-Vincent, «Un public pour la science : l'essor de la vulgarisation au XIX ${ }^{\mathrm{e}}$ siècle », Réseaux, $\mathrm{n}^{\circ}$ 58, 1993, p. 47-66 ; Bernadette Bensaude-Vincent et Anne Rasmussen (dir.), La science populaire dans la presse et l'édition XIX ${ }^{e}$ et XX $X^{e}$ siècles, Paris, Éditions du CNRS, 1997 ; Maurice Crosland, «Popular Science and the Arts : Challenge to Cultural Authority in France under the Second Empire », The British Journal for the History of Science, 34, 2001, p. 301-322. Les premiers ouvrages de vulgarisation de l'astronomie paraissent dès la fin du XVII ${ }^{\mathrm{e}}$ siècle, voir la thèse de Colette Le Lay, Les livres de vulgarisation de l'astronomie (1687-1880), thèse de doctorat dirigée par Jacques Gapaillard et soutenue à l'université de Nantes le 25 mars 2002. Quant au terme de vulgarisation, Auguste Comte l'emploi dans la préface de son Traité philosophique d'astronomie populaire, soulignant la nécessité d'une « sage vulgarisation du système des conceptions astronomiques ».

8. Pour une présentation générale de ce mouvement d'éducation populaire voir Carole Christen, «L'éducation populaire sous la Restauration et la monarchie de Juillet », La Révolution française, 4 (2013), Pédagogies, utopies et révolutions (1789-1848), htttp ://Irf.revues.org/905. Dans le cadre de la présente étude, on écartera les enseignements et ouvrages destinés aux enfants et à la jeunesse 
expéditions lointaines ordonnées par le gouvernement ${ }^{9}$. Entre 1813 et 1847 où il arrête de dispenser ce cours en raison de ses nombreuses activités scientifiques ${ }^{10}$ et politiques $^{11}$, et de sa mauvaise santé, Arago modifie progressivement les finalités de ce cours et l'adapte à un public "populaire" non savant, comme il le précise dans la préface de l'Astronomie populaire - ouvrage en quatre tomes illustrés de 362 figures - issu de ses enseignements mais publié après sa mort ${ }^{12}$ par Jean-Augustin Barral : "l'ouvrage élémentaire que je donne aujourd'hui au public sous le titre d'Astronomie populaire est la reproduction à peu près textuelle du Cours que j'ai fait à l'Observatoire pendant dix-huit années consécutives ${ }^{13}$. " Il est difficile de dater précisément le changement d'orientation de ce cours. Dès le début de sa nomination, il semblerait que les qualités de pédagogue et l'éloquence d'Arago, bien décrites par Cormenin, attirent un public beaucoup plus large que prévu ${ }^{14}$ :

À peine était-il entré en matière qu'il attirait et qu'il concentrait sur lui tous les regards. Le voyez-vous qui prenait pour ainsi dire, la science entre les mains. Il la dépouillait de ses aspérités et des formules techniques et il la rendait si perceptible, que les plus ignorants étaient aussi étonnés et charmés de la comprendre. Sa pantomime expressive animait l'orateur. Il y avait quelque chose de lumineux dans ses démonstrations, et des jets de clarté semblaient sortir de ses yeux, de sa bouche et de ses doigts ${ }^{15}$.

En 1832, Arago demande à l'administrateur du Collège de France de mettre à sa disposition pour son "cours public d'astronomie élémentaire ${ }^{16}$ ", le grand Amphithéâtre car la grande salle de l'Observatoire est devenue insuffisante. Et il obtient ensuite des crédits pour la construction d'un amphithéâtre à l'Observatoire qu'il inaugure lors de l'ouverture de son cours le 15 mai 1841 où il revient sur sa pédagogie :

Je me suis décidé pour des leçons que tout le monde puisse comprendre. Le cours cependant, je vous en avertis, ne sera élémentaire que par la forme. Toutes les

\footnotetext{
9. Maurice Dumas, Arago 1786-1853, la jeunesse de la science, Paris, Belin, 1987 (1 ${ }^{\text {re }}$ éd. : 1943), p. 89.

10. Depuis il 1830 il est secrétaire perpétuel de l'Académie des Sciences (voir Bruno Belhoste, «Arago, les journalistes et l'Académie des Sciences dans les années 1830 », dans Patrick Harismendy (dir.), La France des années 1830 et l'esprit de réforme, Rennes, PUR, coll. « Carnot », p. 253-266) et en 1843 il prend la direction de l'Observatoire.

11. Élu conseiller de la Seine en 1830 et député des Pyrénées-Orientales en juillet 1831 : libéral, opposant dynastique à partir de 1832, il devient républicain en 1840. Après la révolution de février 1848 , il fait partie du gouvernement provisoire de la Seconde République, chargé des ministères de la Marine, de la Guerre et des Colonies.

12. Sous la monarchie de Juillet, certains de ses élèves ont publié, sans l'autorisation d'Arago, les notes qu'ils avaient prises à ses cours : Leçons nouvelles d'astronomie recueillies aux cours publics par un ancien élève de l'École polytechnique, Paris, Baudoins frères, 1826, 210 p. ; Leçons d'astronomie professées à l'Observatoire royal par M. Arago, Paris, Just Rouvier et E. Le Bouvier, 1835, 324 p.

13. François Arago, Astronomie populaire, Paris. L. Guérin, 1967, (1 ${ }^{\text {re }}$ éd. : 1854), t. 1 p. VIII.

14. James Lequeux, François Arago, un savant généreux : physique et astronomie au XIXe siècle, Paris, EDP Sciences, 2008, p. 380.

15. Louis-Marie de Lahaye, vicomte de Cormenin, Livre des orateurs, Paris, Pagnerre, $1842\left(11^{\mathrm{e}}\right.$ éd.), p. 487-488.

16. Lettre du 26 décembre 1832 citée par Jacques Lévy, «Arago et l'astronomie populaire », L'Astronomie, décembre 1986, p. 555.
} 
branches de la science, même les plus délicates, passeront successivement devant vous [...] Sur quel degré de connaissances mathématiques dois-je compter ? Quelle détermination prendre à ce sujet qui puisse convenir à tout le monde ? [...] J'ai pensé, moi, au plus grand nombre, et je me suis décidé pour le parti le plus radical. Je ferai donc le cours sans supposer à mes auditeurs aucune connaissance mathématique quelconque. Les quatre ou cinq propositions élémentaires de géométrie qui nous seront indispensables, je vous les démontrerai, ou du moins j'en fixerai le sens avec précision [...] Le cours sera complet quant au fond, et élémentaire seulement par la forme, par la nature des méthodes adoptées. Ce n'est pas moi qui aurais consenti à dégrader ou même à rétrécir à vos yeux une science dont on dit avec toute raison qu'elle donne la véritable mesure de l'esprit humain [...] Mon unique ambition ici doit être de vous initier aux vérités astronomiques, fruit de trois mille ans d'études, de recherches, de travaux persévérants. Pour arriver à ce but, rien ne me coûtera ; je me répéterai à satiété si cela me paraît nécessaire [...] je consens à vous paraître prolixe, sans élégance, etc. pourvu que vous me trouviez clair et exact dans les démonstrations ${ }^{17}$.

Et dans l'introduction de l'ouverture de son dernier cours d'astronomie le 17 décembre 1846, il émet à nouveau « le vœu que l'auditoire se compose en majorité, même en totalité, de personnes entièrement étrangères aux mathématiques ${ }^{18}$ ". Avec Arago s'affirme l'idée - déjà présente chez Laplace - que la mathématisation n'est pas essentielle à la connaissance scientifique.

On a très peu d'informations sur les auditeurs du cours d'Arago, sur leurs origines sociales, sur leur réceptivité à ces leçons. Jeunes scientifiques, étudiants, savants renommés et bourgeois cultivés viennent nombreux entendre une initiation à l'astronomie ${ }^{19}$. Une gravure parue dans L'Illustration en janvier 1845, montre l'amphithéâtre bondé et au premier plan de l'image sont représentés des hommes et des femmes élégamment habillés debout, faute de places assises libres, quelques-uns discutent entre eux et tournent le dos à l'estrade où se trouve Arago ${ }^{20}$. Ce cours attire la bourgeoise parisienne - hommes et femmes - qui vient chercher dans cet amphithéâtre de l'Observatoire un délassement culturel et mondain. Sur la fresque de Théobald Chartran qui représente en gros plan Arago de profil donnant son cours, les quelques auditeurs qu'on aperçoit portent redingotes pincées et chemises blanches avec cravates plutôt que blouses, tabliers et pantalons à bretelles des ouvriers ou $\operatorname{artisans}^{21}$.

L'ambition d'Arago est de rendre l'astronomie accessible et compréhensible à tous comme en témoigne la simplicité du style des quatre tomes de l'Astronomie

17. François Arago, Astronomie populaire, ouvr. cité, t. 1, « Avertissement de la deuxième édition », p. II-III.

18. Ibid., p. V.

19. François Sarda, Les Arago. François et les autres, Paris, Tallandier, 2002, p. 102.

20. « Cours d'Arago à l'Observatoire de Paris », L'Illustration, Journal universel, 25 janvier 1845, p. 329. Elle est reproduite dans Maurice Dumas, Arago..., ouvr. cité, p. 90-91.

21. On aperçoit aussi un homme d'église et une femme. Ce tableau fait partie de la décoration de l'escalier d'honneur de la Sorbonne peinte par Théobald Chartran entre 1886 et 1889 ; elle est reproduite dans James Lequeux, François Arago, un savant généreux..., ouvr. cité, p. 381. 
populaire qui est bien une transcription de son cours où l'astronome s'adresse ainsi à son auditoire :

Vous voyez que l'explication sur l'inégalité des jours et des nuits est une chose entièrement simple. Nous venons de suivre le soleil depuis le 21 décembre jusqu'au 21 juin, et nous avons vu les jours grandissant toujours. À partir du 21 juin, vous aurez la marche exactement inverse, c'est-à-dire que les jours deviendront de plus en plus courts $[\ldots]$

Vous devez comprendre maintenant pourquoi il y a douze jours de différence entre les pays qui ont adopté la réforme du calendrier et ceux qui ont résisté à cette réforme $[. . .]^{22}$.

L'ouvrage est néanmoins précis et cherche à être exhaustif. Les cinq premiers livres sont consacrés à des notions préliminaires sur la géométrie, la mécanique et l'horlogerie, l'optique, les instruments astronomiques, la vision, qui doivent permettre d'acquérir les connaissances astronomiques développées dans les 29 livres suivants sur la constitution du soleil et des planètes et leurs mouvements propres, sur tous les phénomènes de la voûte étoilée, les saisons et les climats, et le calendrier. Arago mène un combat contre les superstitions et croyances populaires concernant l'influence de la lune sur le climat ou les cultures - la fameuse lune rousse. Il veut aussi apaiser les craintes et les peurs que la soudaineté et la violence de certains événements célestes suscitent, comme les comètes. Détaché de la religion ${ }^{23}$, il ne cherche pas à concilier la réalité des faits expérimentaux avec les Écritures. L'instrumentalisation nécessaire à l'étude du ciel et utilisée dans des expériences célèbres qui ont permis de développer les connaissances astronomiques est souvent représentée - comme la clepsydre de Ctesibius ou le pendule de Foucault - et expliquée très précisément. L'historiographie de la science des astres occupe une place notable dans plusieurs chapitres, témoignant ainsi de l'idée d'un progrès constant. L'astronomie d'observation est bien sûr présente mais c'est la mécanique céleste qui est centrale, ce qui n'est pas surprenant car elle s'est imposée dans la recherche astronomique depuis la fin du XVIII ${ }^{\mathrm{e}}$ siècle et " triomphe $e^{24}$ ".

On ne sait pas si le peuple de Paris - les ouvriers et artisans - se rendait à l'Observatoire pour suivre le cours d'Arago sous la Restauration et la monarchie de Juillet et si plus tard, sous le Second Empire, ils ont lu les quatre volumineux tomes de son Astronomie populaire. Mais on sait que pour Arago, fervent partisan du suffrage universel et d'une nouvelle organisation du travail ${ }^{25}$, l'instruction du peuple, son accès à la connaissance scientifique, sont essentiels car nécessaires à son émancipation par la raison. Arago fait ainsi partie de la commission qui, en 1819, propose au ministre

22. Bibliothèque de l'Observatoire, Manuscrits Arago. Notes prises au cours d'astronomie, $\mathrm{C} 6 / 11,7^{\mathrm{e}}$ leçon, p. 12 et p. 51 .

23. Très attachée à la religion catholique, sa mère n'a pas réussi à lui imposer sa foi.

24. Colette Le Lay, Les livres de vulgarisation de l'astronomie..., ouvr. cité, p. 109.

25. Voir son discours à la Chambre des députés du 16 mai 1840 dans lequel il dénonce les souffrances de la classe ouvrière et y affirme que le progrès social sera obtenu par la réforme électorale : François Sarda, Les Arago..., ouvr. cité, p. 409-418. Huit jours après ce discours 10000 ouvriers viennent à l'Observatoire pour en remercier Arago. Parmi eux, certains ont peut-être suivi son cours d'astronomie. 
Decazes la création de trois chaires d'enseignement au Conservatoire des arts et métiers, permettant ainsi aux ouvriers parisiens de suivre des cours du soir publics et gratuits $^{26}$ et, jusqu'à sa démission en mai 1834, il est un membre actif de son Conseil du perfectionnement ${ }^{27}$. À la fin de la Restauration, il parraine, avec d'autres savants et écrivains, la Bibliothèque populaire ou l'instruction mise à la portée de toutes les classes et de toutes les intelligences, dirigée par Étienne Jean-Baptiste Ajasson de Grandsagne. Il fait également partie, à l'instar de nombreux députés de l'opposition parlementaire - La Fayette, Odilon Barrot, Cormenin, Maugin, Voyer d'Argenson, etc. -, du comité de l'Association libre pour l'éducation du Peuple ${ }^{28}$ née d'une scission de l'Association polytechnique fondée en août 1830 dans le but d'instruire gratuitement les ouvriers parisiens. Son œuvre de vulgarisation de la science astronomique doit être en adéquation avec ses convictions politiques. Ainsi, son cours d'astronomie tel qu'il l'envisage, pénétré par ses idéaux libéraux puis républicains, s'adresse bien aux ouvriers et artisans parisiens même si Arago ne le formule pas clairement contrairement à Auguste Comte qui par son cours d'astronomie cherche à former le peuple à la raison - il qualifie son traité d'astronomie populaire de "philosophique » -, à lui donner une " instruction positive ${ }^{29}$ ".

\section{LE COURS D’ASTRONOMIE ÉLÉMENTAIRE D’AUguSTE COMTE POUR LES OUVRIERS PARISIENS}

Après la révolution de juillet 1830, des anciens élèves de l'École polytechnique fondent l'Association polytechnique ${ }^{30}$ destinée à organiser des cours gratuits pour les ouvriers. Auguste Comte fait partie des signataires de son règlement adopté en août 1830 et est élu - avec trois autres membres - secrétaire de l'Association. Quelques mois plus tard, alors qu'il donne depuis 1826 à l'Athénée Royal un cours de philosophie positive accompagné d'un cours d'astronomie aux " gens du monde », il propose au président de l'Association polytechnique, Victor de Tracy, de « présenter aux ouvriers un cours élémentaire d'astronomie générale, ayant pour principal objet l'exposition raisonnée des phénomènes essentiels du système monde, accompagnée de l'indication des plus importantes applications ${ }^{31}$ ». Il reconnaît qu' " un tel cours

26. Carole Christen, « Les cours pour les ouvriers adultes au Conservatoire des arts et métiers dans le premier XIX ${ }^{\mathrm{e}}$ siècle », Cahiers de RECITS, $\mathrm{n}^{\circ} 10,2014$.

27. Archives administratives du CNAM, Registre du Conseil d'amélioration et de perfectionnement, vol. 1.

28. Archives nationales (AN), F 17/6674, rapport de police du 3 juin 1832 et règlement imprimé de l'Association libre pour l'éducation du peuple, Paris, octobre 1832, p. 14. Cette association républicaine sera liquidée en avril 1834 suite à l'implication de certains de ses membres dans les insurrections parisiennes.

29. «Lettre au maire du $3^{\mathrm{e}}$ arrondissement, vendredi 22 juin 1832 », « Lettre à François Guizot, ministre de l'Instruction publique, 27 novembre 1832 », Auguste Comte, Correspondance et confessions, I, 1814-1840, p. 239-240.

30. François Vatin, «L'Association polytechnique (1830-1900) : "Éducation” ou "Instruction" du peuple? Ou la place des sciences sociales dans la formation du peuple », Management et sciences sociales, $\mathrm{n}^{\circ} 3,2007$, p. 245-296.

31. «Lettre du mardi 14 décembre 1830 au président de l'Association polytechnique », ouvr. cité, p. $218-219$ 
ne puisse être aux ouvriers d'un usage immédiat " mais il affirme que "son utilité n'est pas douteuse, puisqu'il a pour but de leur donner des notions justes et nettes sur un sujet qui, même involontairement, fixe l'attention de tous les hommes, et sur lequel, par conséquent, à défaut d'idées saines, ils en ont nécessairement d'absurdes, qui exercent inévitablement une influence funeste sur le système général de leur intelligence ${ }^{32}$ ". Il est convaincu de l'" aptitude des ouvriers pour un enseignement de cette nature [...] si l'on se dégage des préventions dérivées de nos habitudes sociales ${ }^{33}$ ". Et il espère que ce cours " excitera l'intérêt des ouvriers, et pourra éveiller en eux un juste sentiment de leur dignité par l'attrait que leur offrira un nouvel enseignement essentiellement théorique, exclusivement destiné jusqu'ici à l'usage des messieurs ${ }^{34}$ ". Tous les dimanches à midi, dans la grande salle des Petits-Pères de la mairie du troisième arrondissement, Auguste Comte donne gratuitement un cours de deux heures d'astronomie élémentaire pour les ouvriers parisiens. D'abord très engagé au sein de l'Association polytechnique - il rédige au nom du Comité l'Adresse au Roi des Français dénonçant "l'extrême incurie des chambres, et du ministère pour tout ce qui concerne l'instruction du peuple ; leur dédain pour sa participation aux avantages sociaux en proportion de l'importance de ses travaux ${ }^{35} »-$, il la quitte ${ }^{36}$ et va jusqu'à nier tout lien avec elle pour que son cours d'astronomie ne soit pas suspendu comme les autres cours destinés aux ouvriers suite aux insurrections des 5-6 juin 1832: «la mesure générale prise relativement à la suspension de tous les cours destinés aux ouvriers (mesure que je n'ai point d'ailleurs à discuter ici), ne me concerne spécialement à aucun titre. Mon cours d'astronomie est fait en mon propre et privé nom, sans être rattaché à aucune association quelconque ; c'est ainsi que je l'ai expressément représenté dès l'origine et que mon auditoire l'a toujours conçu ${ }^{37}$. " À l'automne 1832, les cours destinés aux ouvriers sont encore suspendus. Comte s'engage auprès de Guizot à n'introduire aucunes « dissertations politiques dans (son) enseignement [...] dont le caractère exclusivement scientifique était généralement reconnu ${ }^{38}$ ». Il obtient quelques semaines plus tard l'autorisation de rouvrir son cours d'astronomie qu'il poursuit pendant toute la période de la monarchie de Juillet. En 1844, il publie son cours, chacun des 23 chapitres qui composent l'ouvrage " correspond exactement à une leçon orale ${ }^{39}$ ". Comme Arago, il insiste sur l'absence de connaissances mathématiques : "Je me suis essentiellement attaché ici à n'exiger du lecteur aucune préparation mathématique que celle qui résulte communément aujourd'hui d'une première année d'études élémentaires ${ }^{40}$. " Après cent pages de

32. Ibid.

33. Ibid.

34. Ibid.

35. Dans cette adresse, les membres du comité de l'Association polytechnique justifient le soulèvement qui s'est produit la veille à Paris lors de l'annonce du verdict de clémence de la Chambre des Pairs envers les anciens ministres du gouvernement de Charles X, Archives de la Maison Auguste Comte, D. Com.4-2.

36. Dans le premier compte rendu pour l'année 1832, son nom est cité parmi les quatre vice-présidents.

37. « Lettre au maire du $3^{\mathrm{e}}$ arrondissement, vendredi 22 juin 1832 », ouvr. cité, p. 239.

38. « Lettre à François Guizot, mardi 27 novembre 1832 », ouvr. cité, p. 239.

39. Auguste Comte, Traité philosophique d'astronomie populaire ou exposition systématique de toutes les notions de philosophie astronomique, soit scientifiques, soit logiques, qui doivent devenir universellement familières, Paris, Carilian-Goeury et Dalmont, 1844, « préface », p. V.

40. Ibid. 
« discours préliminaire sur l'esprit positif » où il expose la célèbre loi des trois états par lesquels passe l'esprit humain (et les connaissances) - théologique, métaphysique et positif ${ }^{41}$-, suivent quatre parties de cent pages chacune sur l'astronomie positive qui est strictement planétaire limitée aux frontières du système solaire et qui exclut la notion d' " univers " - les astres " extérieurs " (comètes et étoiles) - et nie les récentes découvertes comme la mise en évidence d'une parallaxe stellaire par Bessel en $1838^{42}$. L'astronomie est pour Comte un moyen de promouvoir sa philosophie et par là son projet de réforme sociale ${ }^{43}$ : il ne cherche pas à instruire les ouvriers en leur enseignant les découvertes, les conquêtes et les résultats de la science astronomique comme le fait Arago, mais à former leur esprit en exposant toutes les méthodes et doctrines qui forment, selon lui, les savoirs astronomiques qu'il considère comme la base de la "science de l'humanité ». Ce n'est donc pas le ciel qu'il décrit mais les conceptions successives des astronomes qui s'y sont intéressés, proposant ainsi une approche historique de l'astronomie. Son style est peu limpide et difficilement accessible à tous. Peu d'ouvriers semblent avoir suivi, à ses débuts, son cours d'astronomie. Comte mentionne une lettre « fort remarquable » qui lui a été adressée par un «judicieux ouvrier, imprimeur, auditeur assidu de mon cours ${ }^{44}$ " et dans sa correspondance avec John Stuart Mill, on apprend qu'en 1844 un quart seulement de son auditoire est composé d'ouvriers (horlogers, mécaniciens, imprimeurs) ${ }^{45}$. On sait peu de choses sur cet auditoire d'ouvriers, hormis ce que l'ouvrier menuisier Fabien Magnin (18101884) nous en apprend des années après le cours d'astronomie de Comte qu'il a suivi ${ }^{46}$. Magnin appartient à un petit groupe d'ouvriers qui suit les cours du Conservatoire des arts et métiers et réclame un cours d'astronomie dans cet établissement. Fin avril 1843, l'un d'entre eux découvre le cours de Comte. Magnin et d'autres ouvriers vont alors le suivre assidûment, séduits par les questions philosophiques, politiques et sociales posées par Comte. En janvier 1846, ils font imprimer à leurs frais une annonce de ce cours qu'ils distribuent dans tous les ateliers parisiens, ce qui, d'après Magnin, amène à son cours un assez grand nombre d'ouvriers ${ }^{47}$.

41. Il reprend les grandes lignes de son Cours de philosophie positive rédigé de 1830 à 1842 .

42. Ce qui permet à Bessel de déterminer les premières mesures précises de la distance d'une étoile fixe.

43. Philosophes et sociologues s’y sont intéressé : Bernadette Bensaude-Vincent : « Auguste Comte, la science populaire d'un philosophe », Corpus. Revue de philosophie, n 4, 1987, p. 143-167 ; « L'astronomie populaire, priorité philosophique et projet politique », Revue de synthèse, $\mathrm{n}^{\circ}$ 1, janv.-mars 1991, p. 49-59 ; "Comte et la diffusion des sciences », dans Annie Petit (dir.), Auguste Comte, trajectoires positivistes, 1798-1998, Paris, L'Harmattan, 2003, p. 127-134 ; Annie Petit, « la diffusion des savoirs comme devoir positiviste », Romantisme, 1989, n 65, p. 7-26 ; François Vatin, « Auguste Comte, les sciences d'application et la formation du peuple », Revue philosophique de la France et de l'étranger, 2007/4 Tome 132, p. 421-435.

44. Auguste Comte, Traité philosophique d'astronomie populaire..., ouvr. cité, p. 222.

45. « Lettre à John Stuart Mill, mercredi $1^{\mathrm{er}}$ mai 1844 », Auguste Comte, Correspondance et confessions, II, 1841-1845, p. 247.

46. Fabien Magnin, «Discours à l'occasion du $21^{\mathrm{e}}$ anniversaire de la mort d'Auguste Comte, 5 septembre 1878 », Études sociales, Paris, 1913, p. 126-141. Voir sa notice biographique dans Geneviève Poujol, Madeleine Romer, Dictionnaire biographique des militants. XIX $-X X^{e}$ siècles. De l'éducation populaire à l'action culturelle, Paris, L'Harmattan, 1996, p. 253-254.

47. Les ouvriers sont majoritaires au sein de la Société positiviste que fonde Comte le 8 mars 1848 , après avoir transformé, en janvier 1848, son cours d'astronomie en « cours philosophique sur l'histoire générale de l'Humanité ». Peu avant sa mort, en 1857, Comte désigne Fabien Magnin comme son successeur 
Quand trente ans plus tard, en 1878, il évoque cette période, Magnin n'ignore plus rien de la doctrine positiviste, il rappelle alors le rôle de l'astronomie dans l'économie générale des savoirs pour les positivistes :

Auguste comte [...] savait très bien que l'astronomie avait été le grand moteur qui, en transformant l'esprit des populations, les avait amenées à l'état rationnel où nous les voyons ; et que les principaux progrès dont nous jouissons, ont pour base la diffusion des principes généraux de l'astronomie parmi les populations, où elle fait épanouir ce qu'il y a de germes d'aptitude théorique sans les cerveaux de chacun. Enfin, Auguste Comte savait très bien que l'astronomie est le meilleur moyen pour reconnaître parmi les esprits propres à l'abstraction ceux qui sont capables d'aborder franchement les difficiles problèmes de sociologie et de morale, et pour distinguer, parmi eux, ceux qui sont à la fois assez modestes et assez énergiques pour s'astreindre d'eux-mêmes à n'affirmer jamais ce qui est exactement connu ${ }^{48}$.

À côté de ces ambitieux cours et traités d'astronomie populaire d'Arago et de Comte, d'autres enseignements et publications plus modestes sont proposés au peuple $^{49}$, témoignant ainsi de la place accordée à cette science dans l'éducation populaire au cours du premier XIX ${ }^{\mathrm{e}}$ siècle.

\section{L'ASTRONOMIE DANS L'ÉDUCATION POPULAIRE}

Charles Dupin ${ }^{50}$, "Professeur des ouvriers » comme il se désigne lui-même depuis sa chaire de géométrie et de mécanique appliquées aux arts du Conservatoire des arts et métiers, consacre le discours d'ouverture de son cours, en novembre 1826, aux effets de l'enseignement populaire. Pour justifier l'utilité de l'enseignement des "vérités mathématiques" aux "simples ouvriers », il montre leur importance dans la compréhension du "système du monde ": " en peu de mots, je pourrais, ce me semble, rendre clair cet admirable système du monde, qu'il a fallu cinq mille ans pour découvrir et calculer ${ }^{51}$ ». Par les exemples très concrets qu'il donne dans la suite de son discours, il entend par «système du monde » l'astronomie bien qu'il n'emploie pas ce terme : " nous ferons aisément comprendre aux ouvriers la grandeur de notre système solaire et celle des masses qui le composent, et l'ordre si simple, si beau, et j'ose dire si divin, des mouvements éternels qui en règlent les phénomènes ${ }^{52}$. " Il compare les

à la Société positiviste. Sous le Second Empire, Magnin enseigne à son domicile l'astronomie et la géométrie et il participe à la renaissance du mouvement ouvrier.

48. Fabien Magnin, Études sociales, ouvr. cité, p. 129-130.

49. En revanche, le cours public et gratuit d'astronomie que Frédéric Petit donne à l'Observatoire de Toulouse à partir de 1838 s'adresse clairement « aux gens du monde »; sa démarche - et ses objectifs didactiques - s'inscrit dans le mouvement initié par son maître Arago, mais elle s'en écarte nettement quant à l'assistance attendue, voir Jérôme Lamy, L'Observatoire de Toulouse aux XVIII et XIX ${ }^{e}$ siècles, Rennes, PUR, 2007, p. 293-294.

50. Carole Christen, François Vatin (dir.), Charles Dupin (1784-1873). Ingénieur, savant, économiste, pédagogue et parlementaire du Premier au Second Empire, Presses Universitaires de Rennes, coll. « Carnot », 2009.

51. Charles Dupin, Effets de l'enseignement populaire de la lecture, de l'écriture, de l'arithmétique, de la géométrie et de la méchanique appliquée aux arts, sur les prospérités de la France, Paris, 1826, p. 4.

52. Ibid., p. 5. 
forces du système du monde aux forces dont on peut disposer sur la terre, comme la force du cheval traînant une voiture sur une route du cheval : "Pour produire une quantité d'action comparable à celle du simple mouvement de la terre autour du soleil, il faudrait attacher au char de la terre plus de dix milliards d'attelages ayant chacun dix milliards de chevaux. Pour faire parcourir au soleil le même espace qu'à la terre, dans un même temps, il faudrait 1348460 fois dix milliards de chevaux ${ }^{53}$. » Par ces quelques exemples, Dupin, qui n'est pas astronome, se targue de pouvoir enseigner des rudiments de la science astronomique : "De nos jours, avec des moyens bien simples, des démonstrations bien aisées et des calculs élémentaires, on peut faire comprendre aux artisans de toutes les professions ces vérités scientifiques et ces secrets du système du monde que les philosophes de l'Antiquité, les plus illustres par leur génie, ont cherchés sans pouvoir les découvrir ${ }^{54}$. "Ils doivent alors permettre de faire des rapprochements entre les forces du système du monde et les forces sur la terre et renforcer la croyance religieuse en un "Créateur de toutes choses ", un "Créateur de cette harmonie éternelle », au "Tout-Puissant»: "Quand les ouvriers de l'industrie viendront à comparer la force du bras le plus robuste à la force qu'il faut pour mouvoir la terre, le soleil et les planètes, les satellites et les comètes, ils comprendront qu'une intelligence supérieure, immortelle, infinie, veille à la géométrie du monde, et règle, par les lois d'une science immuable, les formes et les mouvements de l'univers ${ }^{55}$. " Le poids de l'Église sur les mentalités est considérable, et Dupin n'est pas le seul à réduire l'astronomie à la simple admiration des beautés du Ciel prodiguées à l'homme par un Dieu toujours soucieux d'en faire le centre de sa Création. L'astronomie doit être compatible avec les Écritures, elle peut être utilisée comme un moyen de servir la religion et de renforcer la croyance en Dieu. À la même époque, suivant l'exemple de Dupin au Conservatoire des arts et métiers, Claude-Lucien Bergery fonde en 1825 à Metz des cours publics pour ouvriers ${ }^{56}$, d'arithmétique appliquée aux spéculations commerciales et industrielles, de géométrie appliquée à l'industrie, d'économie industrielle et d'astronomie. C'est sur la demande des ouvriers messins qu'il aurait fait ce cours d'astronomie : " ce sont vos sollicitations réitérées qui m'ont porté à l'entreprendre. [...] Avides d'instruction, encouragés par vos succès dans l'étude des sciences, vous m'avez exprimé, à diverses reprises, le vif désir de bien connaître ce merveilleux univers ${ }^{57}$. " Il y définit l'astronomie comme "les lois éternelles imposées à ces astres [Soleil, Lune, Étoiles] et à la Terre, par le créateur de l'univers, jointes aux faits qui en découlent ${ }^{58}$ ". L'ouvrage qu'il présente comme une "simple description " appuyée sur des "notions exactes » est composé de neuf chapitres courts (l'ouvrage fait 150 pages) sur le soleil, la terre, la lune, les éclipses, les planètes, les comètes, les étoiles, le calendrier et les cadrans solaires. L'histoire de l'astronomie y est absente

53. Ibid., p. 5-6.

54. Ibid., p. 6.

55. Ibid., p. 7.

56. François Vatin, Morale industrielle et calcul économique dans le premier XIXe siècle. L'économie industrielle de Claude-Lucien Bergery (1787-1863), Paris, L'Harmattan, 2007, chapitre II, p. 49-127.

57. Claude-Lucien Bergery, Astronomie élémentaire ou description géométrique de l'univers faite aux ouvriers messins, Metz, Thiel, 1832, « dédicace ».

58. Ibid., p. 7. 
mais on trouve quelques formules mathématiques et des données chiffrées. Pour Bergery, l'astronomie doit permettre de combattre les préjugés des ouvriers tout en renforçant leur foi religieuse car la compréhension du système du monde, considéré par Bergery, à l'instar de la grande majorité de ses contemporains, comme étant la création de Dieu, doit les rendre admiratifs du Créateur : "je souhaite que les notions exactes qu'elle [cette simple description] vous donnera sur les phénomènes, la structure et l'harmonie de l'œuvre immense, infinie du Créateur, redressent vos idées, détruisent les préjugés dont votre enfance a été imbue, vous pénètrent d'admiration et vous rendent religieux comme doivent l'être des hommes éclairés et libres ${ }^{59}$. $\mathrm{On}$ peut y voir comme une tentative de rechristianisation du peuple - des ouvriers - par l'enseignement de l'astronomie qui doit aussi combattre leur obscurantisme, leurs croyances superstitieuses :

Longtemps l'astrologie ou l'art de lire dans les astres les destinées de l'individu et des nations fut en honneur, quoiqu'elle ne reposât que sur de vagues conjectures et de bizarres assertions. Grâce aux lumières de la vraie religion, aux progrès de la vraie science et aux observations du sens commun, l'astrologie fut appréciée enfin comme elle devait l'être et le mépris général vint faire justice des jongleries de quelques imposteurs. Telle a été néanmoins la profondeur, l'universalité du mal, qu'il en reste des traces encore, que nos campagnes sont infectées d'une superstition aussi impie que honteuse, et qu'il est nécessaire encore de songer aux moyens de l'extirper. L'unique moyen de parvenir à ce but n'est pas l'enseignement de la religion, n'est pas celui de la raison, c'est celui du fait, de l'astronomie telle que le peuple lui-même peut la comprendre, l'explication toute naturelle de l'univers, du mouvement des astres, des conjonctures où ils peuvent se trouver. C'est un enseignement qui est désormais inséparable de celui de la cosmographie générale. Il est une autre superstition non moins irréligieuse et non moins honteuse que celle-là, c'est la foi à l'almanach, c'est la croyance populaire aux prédictions de pluie et de beau temps du calendrier. Honte à ceux qui abusent si effrontément de la crédulité des simples et spéculent d'une manière si ignoble sur les préjugés du peuple ; mais compassion pour les victimes de cette cupidité, compassion active, portant secours aux malheureux et remède au mal ${ }^{60}$.

L'astronomie, et la science en général, sont loin d'être ressenties comme des menaces pour la religion comme elles le seront à la fin du XIX ${ }^{\mathrm{e}}$ siècle avec les militants laïcs qui dénient à l'Église la moindre compétence scientifique et combattent la «science théologique ». Comme d'autres savoirs, l'astronomie doit participer à la formation intellectuelle de l'ouvrier, à son émancipation et son ascension sociale et, plus généralement, à l'harmonie sociale qui est remise en cause par les premières grandes agitations politiques et sociales que connaît la monarchie de Juillet en 1831 et 1832. C'est l'idée que le progrès de la diffusion des sciences peut réconcilier toutes les classes sociales :

59. Ibid., « dédicace ».

60. Nouveau manuel des écoles primaires, moyennes et normales, ou guide complet des instituteurs et des institutrices, Paris, A la librairie encyclopédique de Roret, 1836, p. 124. 
J'espère donc bien qu'un jour il [l'ouvrier] aura part entière à la vie intellectuelle, qu'il en connaîtra tout le charme, et qu'il trouvera dans les ressources, dans la sagesse de son esprit, les moyens d'assurer son bien-être. Alors, et cette époque n'est peut-être pas très loin de nous, alors cesseront les convulsions sociales, parce qu'il y a aura liberté sage et nivellement de haute civilisation : les ouvriers et les maîtres, les pauvres et les riches seront à peu près égaux en savoir et en moralité, comme ils l'étaient en ignorance et en vices, dans les temps de servitude et de barbarie $^{61}$.

L'astronomie est aussi présente dans les collections et encyclopédies populaires qui paraissent à la fin de la Restauration ou sous la monarchie de Juillet et dont la lecture est recommandée par la presse ouvrière ${ }^{62}$, telles l'Encyclopédie Roret ${ }^{63}$ qui publie un Manuel d'Astronomie ou Traité Élémentaire de cette Science, d'après l'état actuel de nos connaissances ${ }^{64}$, et la Bibliothèque populaire ou l'instruction mise à la portée de toutes les classes et de toutes les intelligences qui consacre parmi la centaine de titres qu'elle contient en 1833 un volume à un Traité élémentaire d'astronomie ${ }^{65}$. Ces deux collections figurent dans la liste des ouvrages qui peuvent servir à la formation de bibliothèques populaires proposée par François Delessert en $1836^{66}$. En 1846, l'éditeur Henri-Léon Curmer souhaite que les sciences puissent être transmises à tous par les bibliothèques populaires, ces dernières doivent servir de relais à la vulgarisation scientifique, soulignant la nécessité de diffuser en province le cours d'astronomie d'Arago :

N'est-il pas vrai de dire qu'un cours spécial d'astronomie élémentaire, professé à Paris par M. Arago, et envoyé gratis à toutes les bibliothèques communales, pour être lu dans les écoles primaires du royaume, atteindrait le véritable but que se propose le gouvernement en fondant des cours, et l'illustre professeur en y recherchant la gloire qu'il est en droit d'attendre?

Si la science doit travailler courageusement à repousser les ténèbres qui l'entourent, elle ne doit par marcher seule, et laisser ignorer ses traces à ceux qui sont en état de la suivre, il faut que le lien soit constant entre tous les degrés de la science, et que le chemin soit rendu accessible à toutes les intelligences ${ }^{67}$.

61. Claude-Lucien Bergery, Astronomie élémentaire..., ouvr. cité, « dédicace ».

62. L'Écho de la Fabrique y consacre plusieurs articles en $1833: \mathrm{n}^{\circ} 11$, p. $90 ; \mathrm{n}^{\circ} 14$, p. $113 ; \mathrm{n}^{\circ} 23$, p. $190 ; n^{\circ} 24 ; n^{\circ} 31$.

63. Anne-Françoise Garçon, «Innover dans le texte. L'Encyclopédie Roret et la vulgarisation des techniques, 1830-1880 », Les Archives de l'invention, Colloque CNAM Paris, novembre 2004, site HALSHS du CNRS.

64. Encyclopédie RORET, Manuel d'Astronomie ou Traité Élémentaire de cette Science, d'après l'état actuel de nos connaissances contenant L'Exposé complet du système du monde, basé sur les travaux les plus récents et les résultats qui dérivent des recherches de M. Pouillet sur la température du soleil, et de celles de M. Arago sur la densité de la partie extérieure de cet astre, Paris, Roret, Libraire, 1825, $256 \mathrm{p}$.

65. Pour une présentation de cet ouvrage voir Colette Le Lay, Les Livres de vulgarisation de l'astronomie..., ouvr. cité, p. 134-136.

66. Carole Christen, «Les bibliothèques populaires : un remède à la question sociale dans la première moitié du XIX ${ }^{\mathrm{e}}$ siècle? ", dans Agnès Sandras (dir.), Des bibliothèques populaires à la lecture publique, Villeurbannes, Presses de l'ENSSIB, 2014, p. 53-76.

67. Henri-Léon Curmer, De l'établissement des bibliothèques communales en France, Paris, Guillaumin et C ${ }^{\text {ie }}, 1846$, p. 92. 
Sans doute l'astronomie populaire enseignée par François Arago dans l'amphithéâtre de l'Observatoire et celle enseignée par Auguste Comte dans une salle de la mairie du troisième arrondissement sont-elles bien différentes dans leur forme, leur contenu, leurs objectifs et donnent-elles à voir une science astronomique bien différente - on est loin chez Comte de l'astronomie conquérante qui recule les frontières du connu. Pour autant, Arago et Comte ${ }^{68}$ ont l'un et l'autre joué un rôle essentiel dans l'émergence de la vulgarisation scientifique qui s'épanouit sous le Second Empire et atteint son apogée dans les années 1880 avec la célèbre Astronomie populaire de Camille Flammarion qui, une quinzaine d'années auparavant, avait commencé à populariser l'astronomie en s'adressant à deux publics nettement séparés : l'un, composé d'ouvriers et d'apprentis de tous âges se pressant en foule dans un amphithéâtre de l'école Turgot, l'autre, constitué par un public mondain de bourgeois cultivés louant d'avance ses places à la salle des conférences du boulevard des Capucines ${ }^{69}$.

(Université de Lille 3 et IUF)

68. Dans la deuxième moitié du XIX ${ }^{\mathrm{e}}$ siècle, d'autres réformateurs sociaux et penseurs politiques ont aussi accordé une place importante à l'astronomie dans leur œuvre d'éducation du peuple comme Jean Reynaud, sous secrétaire d'État à l'Instruction publique en 1848, qui écrit en 1854, Philosophie religieuse. Terre et ciel; et Auguste Blanqui, le révolutionnaire socialiste, surnommé " l'Enfermé », qui publie en 1872, L'éternité par les astres (réédité en 2012 avec une préface de Jacques Rancière).

69. Stéphane Douailler, «Des élèves pour l'astronomie », dans Les sauvages dans la cité. Autoémancipation du peuple et instruction des prolétaires au XIX siècle, Paris, Champ Vallon, 1985, p. 58 et Robert Fox, « Les conférences mondaines sous le Second Empire », Romantisme, 1989, n 65, p. 49-57. 